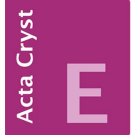
COMMUNICATIONS

ISSN 2056-9890

\title{
Crystal structure of 4-chloro-2-\{(E)-[(3,4- dimethylphenyl)imino]methyl\}phenol
}

\author{
Muhammad Salim, ${ }^{\text {a }}$ Muhammad Nawaz Tahir, ${ }^{\text {b* }}$ \\ Munawar Ali Munawar, ${ }^{a}$ Muhammad Shahid ${ }^{\mathrm{a}}$ and \\ Hazoor Ahmad Shad ${ }^{\mathrm{c}}$
}

a Department of Chemistry, University of the Punjab, Lahore, Punjab, Pakistan, ${ }^{\mathbf{b}}$ Department of Physics, University of Sargodha, Sargodha, Punjab, Pakistan, and 'Department of Chemistry, University of Sargodha, Sargodha, Punjab, Pakistan.

*Correspondence e-mail: dmntahir_uos@yahoo.com

Received 10 May 2015; accepted 16 May 2015

Edited by W. T. A. Harrison, University of Aberdeen, Scotland

In the title compound, $\mathrm{C}_{15} \mathrm{H}_{14} \mathrm{ClNO}$, which is isostructural with its bromo analogue [Tahir et al. (2012). Acta Cryst., E68, o2730], the dihedral angle between the planes of the aromatic rings is $2.71(7)^{\circ}$ and an intramolecular $\mathrm{O}-\mathrm{H} \cdots \mathrm{N}$ hydrogen bond closes an $S(6)$ ring. In the crystal, extremely weak C$\mathrm{H} \cdots \pi$ interactions link the molecules into a three-dimensional network.

Keywords: crystal structure; phenol; intramolecular hydrogen bonding; $\mathrm{C}-\mathrm{H} \cdots \pi$ interactions.

CCDC reference: 1401503

\section{Related literature}

For related structures, see: Demircioğlu et al. (2014); Jin et al. (2012); Sun et al. (2013); Tahir et al. (2012).

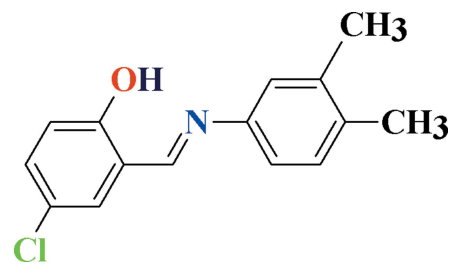

\section{Experimental}

\subsection{Crystal data}

$\mathrm{C}_{15} \mathrm{H}_{14} \mathrm{ClNO}$

$M_{r}=259.72$

Monoclinic, $P 2_{1} / n$

$a=12.1875(10) \AA$

$b=7.4438(5) \AA$

$$
\begin{aligned}
& c=14.3141(12) \AA \\
& \beta=101.549(4)^{\circ} \\
& V=1272.30(17) \AA^{3} \\
& Z=4 \\
& \text { Mo } K \alpha \text { radiation }
\end{aligned}
$$

$\mu=0.29 \mathrm{~mm}^{-1}$

$T=296 \mathrm{~K}$

$0.25 \times 0.20 \times 0.14 \mathrm{~mm}$

\subsection{Data collection}

Bruker Kappa APEXII CCD diffractometer

Absorption correction: multi-scan (SADABS; Bruker, 2007)

$T_{\min }=0.933, T_{\max }=0.968$

2.3. Refinement

$R\left[F^{2}>2 \sigma\left(F^{2}\right)\right]=0.041$

$w R\left(F^{2}\right)=0.116$

$S=1.04$

2785 reflections

166 parameters

$\mathrm{H}$-atom parameters constrained

$\Delta \rho_{\max }=0.24{\mathrm{e} \AA^{-3}}^{-3}$

$\Delta \rho_{\min }=-0.22 \mathrm{e} \AA^{-3}$

\begin{tabular}{|c|c|c|c|c|}
\hline$D-\mathrm{H} \cdots A$ & $D-\mathrm{H}$ & $\mathrm{H} \cdots A$ & $D \cdots A$ & $D-\mathrm{H} \cdots A$ \\
\hline $\mathrm{O} 1-\mathrm{H} 1 \cdots \mathrm{N} 1$ & 0.82 & 1.87 & $2.5998(19)$ & 149 \\
\hline $\mathrm{C} 3-\mathrm{H} 3 \cdots C g 1^{\mathrm{i}}$ & 0.93 & 2.98 & $3.732(2)$ & 139 \\
\hline $\mathrm{C} 6-\mathrm{H} 6 \cdots C g 2^{\mathrm{ii}}$ & 0.93 & 2.93 & $3.576(2)$ & 128 \\
\hline $\mathrm{C} 14-\mathrm{H} 14 B \cdots C g 2^{\mathrm{iii}}$ & 0.96 & 2.96 & $3.656(2)$ & 131 \\
\hline
\end{tabular}

Table 1

Hydrogen-bond geometry $\left(\AA,^{\circ}\right)$.

$\mathrm{Cg} 1$ and $\mathrm{Cg} 2$ are the centroids of the $\mathrm{C} 1-\mathrm{C} 6$ and $\mathrm{C} 8-\mathrm{C} 13$ benzene rings, respectively.

Data collection: APEX2 (Bruker, 2007); cell refinement: SAINT (Bruker, 2007); data reduction: $S A I N T$; $\operatorname{program}(\mathrm{s})$ used to solve structure: SHELXS97 (Sheldrick, 2008); program(s) used to refine structure: SHELXL2014 (Sheldrick, 2015); molecular graphics: ORTEP-3 for Windows (Farrugia, 2012) and PLATON (Spek, 2009); software used to prepare material for publication: WinGX (Farrugia, 2012) and PLATON.

\section{Acknowledgements}

The authors acknowledge the provision of funds for the purchase of the diffractometer and encouragement by Dr Muhammad Akram Chaudhary, Vice Chancellor, University of Sargodha, Pakistan.

Supporting information for this paper is available from the IUCr electronic archives (Reference: HB7424).

\section{References}

Bruker (2007). APEX2, SAINT and SADABS. Bruker AXS Inc., Madison, Wisconsin, USA.

Demircioğlu, Z., Albayrak, C., Çiğdem, \& Büyükgüngör, O. (2014). J. Mol. Struct. 1065-1066, 210-222.

Farrugia, L. J. (2012). J. Appl. Cryst. 45, 849-854.

Jin, Y.-B., Chang, Y.-K., Zhang, Y. \& Lei, K.-W. (2012). Acta Cryst. E68, o2415.

Sheldrick, G. M. (2008). Acta Cryst. A64, 112-122.

Sheldrick, G. M. (2015). Acta Cryst. C71, 3-8.

Spek, A. L. (2009). Acta Cryst. D65, 148-155.

Sun, L.-X., Zhu, L.-Z. \& Wang, J.-K. (2013). Acta Cryst. E69, o631.

Tahir, M. N., Khan, A. H., Tariq, M. I., Hussain, I. \& Shafiq, M. (2012). Acta Cryst. E68, o2730. 


\section{supporting information}

Acta Cryst. (2015). E71, o416 [doi:10.1107/S2056989015009354]

\section{Crystal structure of 4-chloro-2-\{(E)-[(3,4-dimethylphenyl)imino]methyl\}phenol}

Muhammad Salim, Muhammad Nawaz Tahir, Munawar Ali Munawar, Muhammad Shahid and Hazoor Ahmad Shad

\section{S1. Comment}

The title compound, (I, Fig. 1) has been synthesized in continuation of forming different derivatives of 3,4-dimethylaniline. (I) will also be utilized for synthesizing different metal complexes.

The crystal structures of 4-bromo-2-((E)-[(3,4-dimethylphenyl)imino]methyl)phenol (Tahir et al., 2012), 2-((3,4-dimethylphenyl)carbonoimidoyl)-3-methoxyphenol (Demircioğlu et al., 2014), $N$-[(E)-4-bromobenzylidene]-3,4-dimethylaniline (Sun et al., 2013) and $N$-[(E)-4-fluorobenzylidene]-3,4-dimethylaniline (Jin et al., 2012) have been published which are related to the title compound.

The title compound is isostructural to 4-bromo-2-((E)-[(3,4-dimethyl phenyl)imino]methyl)phenol (Tahir et al., 2012) and is almost planar with r. m. s. deviation of $0.0325 \AA$, with maximum deviation of 0.0803 (9) $\AA$ for $\mathrm{Cl1}$ atom from the mean square plane. There exist intramolecular H-bonding of $\mathrm{O}-\mathrm{H} \cdots \mathrm{N}$ type (Table 1, Fig. 1) with $S(6)$ ring motif. There exist $\mathrm{C}-\mathrm{H} \cdots \pi$ interactions (Table 1 ).

\section{S2. Experimental}

Equimolar quantities of 5-chlorosalicylaldehyde and 3,4-dimethylaniline were refluxed in methanol for $3 \mathrm{~h}$. The solution was kept at room temperature for crystallization which affoarded light yellow plates after $72 \mathrm{~h}$.

\section{S3. Refinement}

The $\mathrm{H}$ atoms were positioned geometrically $(\mathrm{C}-\mathrm{H}=0.93-0.96 \AA, \mathrm{O}-\mathrm{H}=0.82 \AA)$ and refined as riding with $U_{\text {iso }}(\mathrm{H})=$ $x U_{\text {eq }}(\mathrm{C}, \mathrm{O})$, where $x=1.5$ for methyl \& hydroxy and $x=1.2$ for other $\mathrm{H}$-atoms. 


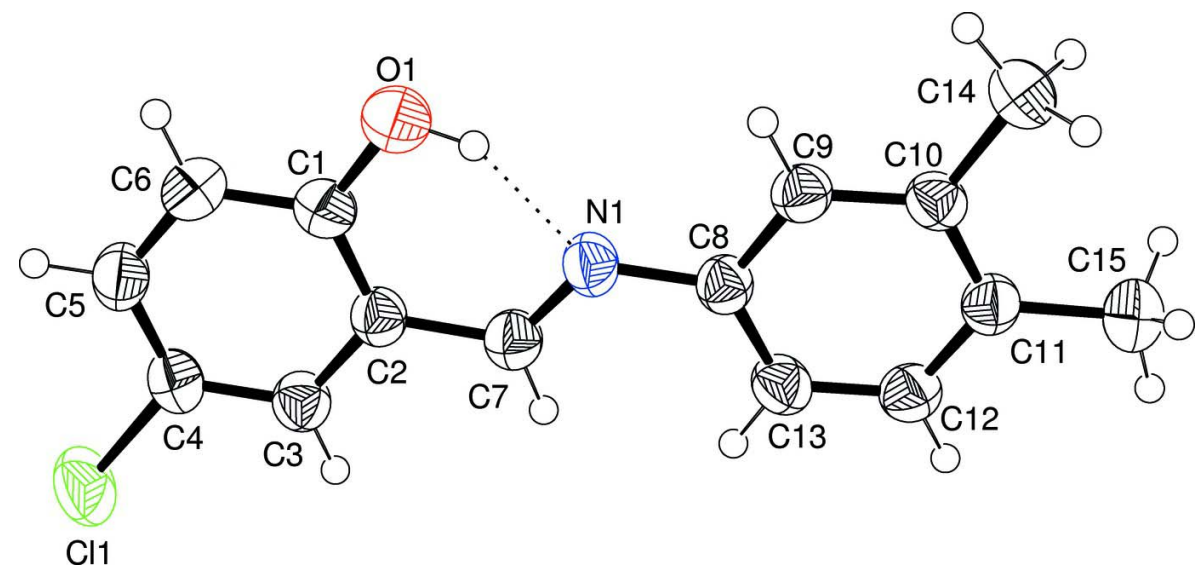

\section{Figure 1}

View of the title compound with displacement ellipsoids drawn at the $50 \%$ probability level. The dotted line shows intramolecular H-bonding.

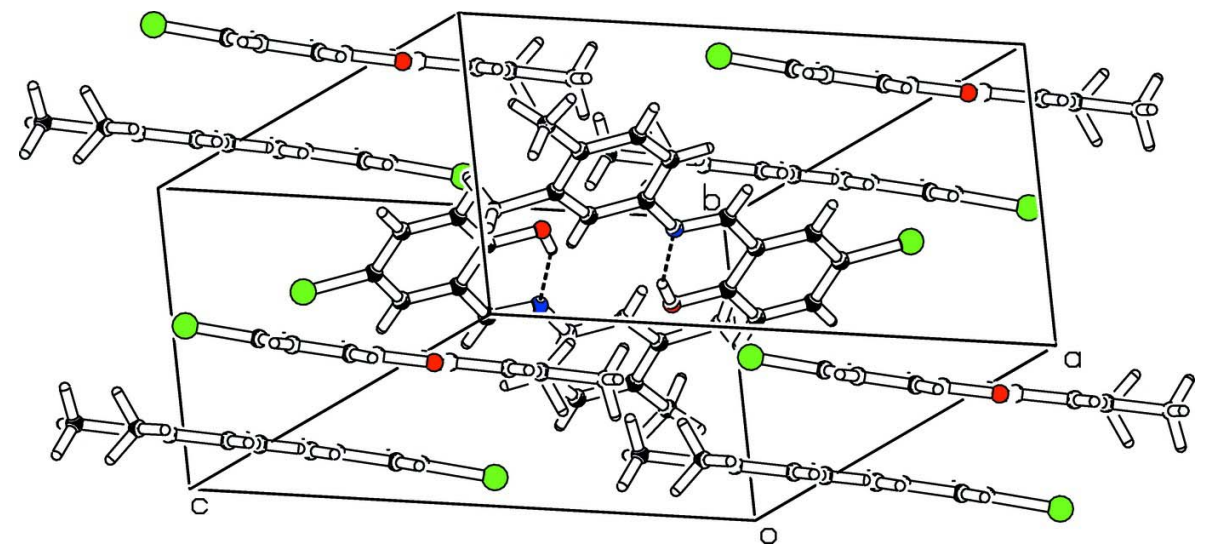

\section{Figure 2}

Packing diagram for the title compound.

\section{4-Chloro-2-\{(E)-[(3,4-dimethylphenyl)imino]methyl $\}$ phenol}

\section{Crystal data}

\section{$\mathrm{C}_{15} \mathrm{H}_{14} \mathrm{ClNO}$}

$M_{r}=259.72$

Monoclinic, $P 2{ }_{1} / n$

$a=12.1875(10) \AA$

$b=7.4438(5) \AA$

$c=14.3141(12) \AA$

$\beta=101.549(4)^{\circ}$

$V=1272.30(17) \AA^{3}$

$Z=4$

\section{Data collection}

Bruker Kappa APEXII CCD diffractometer

Radiation source: fine-focus sealed tube Graphite monochromator
$F(000)=544$

$D_{\mathrm{x}}=1.356 \mathrm{Mg} \mathrm{m}^{-3}$

Mo $K \alpha$ radiation, $\lambda=0.71073 \AA$

Cell parameters from 1871 reflections

$\theta=2.0-27.0^{\circ}$

$\mu=0.29 \mathrm{~mm}^{-1}$

$T=296 \mathrm{~K}$

Plate, light yellow

$0.25 \times 0.20 \times 0.14 \mathrm{~mm}$

Detector resolution: 7.80 pixels $\mathrm{mm}^{-1}$

$\omega$ scans

Absorption correction: multi-scan

(SADABS; Bruker, 2007) 
$T_{\min }=0.933, T_{\max }=0.968$

10293 measured reflections

2785 independent reflections

1871 reflections with $I>2 \sigma(I)$

$R_{\text {int }}=0.024$

\section{Refinement}

Refinement on $F^{2}$

Least-squares matrix: full

$R\left[F^{2}>2 \sigma\left(F^{2}\right)\right]=0.041$

$w R\left(F^{2}\right)=0.116$

$S=1.04$

2785 reflections

166 parameters

0 restraints

Primary atom site location: structure-invariant direct methods

$$
\begin{aligned}
& \theta_{\text {max }}=27.0^{\circ}, \theta_{\text {min }}=2.0^{\circ} \\
& h=-15 \rightarrow 12 \\
& k=-8 \rightarrow 9 \\
& l=-18 \rightarrow 15
\end{aligned}
$$

Special details

Geometry. All e.s.d.'s (except the e.s.d. in the dihedral angle between two 1.s. planes) are estimated using the full covariance matrix. The cell e.s.d.'s are taken into account individually in the estimation of e.s.d.'s in distances, angles and torsion angles; correlations between e.s.d.'s in cell parameters are only used when they are defined by crystal symmetry. An approximate (isotropic) treatment of cell e.s.d.'s is used for estimating e.s.d.'s involving 1.s. planes.

Refinement. Refinement of $F^{2}$ against ALL reflections. The weighted $R$-factor $w R$ and goodness of fit $S$ are based on $F^{2}$, conventional $R$-factors $R$ are based on $F$, with $F$ set to zero for negative $F^{2}$. The threshold expression of $F^{2}>\sigma\left(F^{2}\right)$ is used only for calculating $R$-factors $(\mathrm{gt})$ etc. and is not relevant to the choice of reflections for refinement. $R$-factors based on $F^{2}$ are statistically about twice as large as those based on $F$, and $R$-factors based on ALL data will be even larger.

Fractional atomic coordinates and isotropic or equivalent isotropic displacement parameters $\left(\AA^{2}\right)$

\begin{tabular}{lllll}
\hline & $x$ & $y$ & $z$ & $U_{\text {iso }} * / U_{\text {eq }}$ \\
\hline C11 & $0.31558(4)$ & $0.47763(8)$ & $0.94032(3)$ & $0.0702(2)$ \\
O1 & $0.45252(11)$ & $0.6419(2)$ & $0.58212(9)$ & $0.0684(4)$ \\
H1 & 0.4036 & 0.6151 & 0.5361 & $0.103^{*}$ \\
N1 & $0.26240(12)$ & $0.51118(17)$ & $0.49303(10)$ & $0.0442(4)$ \\
C1 & $0.41674(14)$ & $0.6064(2)$ & $0.66249(12)$ & $0.0462(4)$ \\
C2 & $0.31233(14)$ & $0.5273(2)$ & $0.66205(11)$ & $0.0400(4)$ \\
C3 & $0.28167(14)$ & $0.4894(2)$ & $0.74847(12)$ & $0.0434(4)$ \\
H3 & 0.2128 & 0.4363 & 0.7492 & $0.052^{*}$ \\
C4 & $0.35292(14)$ & $0.5305(2)$ & $0.83271(12)$ & $0.0447(4)$ \\
C5 & $0.45476(15)$ & $0.6104(2)$ & $0.83316(12)$ & $0.0499(4)$ \\
H5 & 0.5022 & 0.6383 & 0.8907 & $0.060^{*}$ \\
C6 & $0.48620(15)$ & $0.6486(2)$ & $0.74880(13)$ & $0.0524(5)$ \\
H6 & 0.5549 & 0.7035 & 0.7493 & $0.063 *$ \\
C7 & $0.23682(15)$ & $0.4821(2)$ & $0.57324(12)$ & $0.0432(4)$ \\
H7 & 0.1679 & 0.4304 & 0.5753 & $0.052^{*}$ \\
C8 & $0.18970(13)$ & $0.4694(2)$ & $0.40504(11)$ & $0.0394(4)$ \\
C9 & $0.23021(14)$ & $0.5050(2)$ & $0.32363(12)$ & $0.0414(4)$ \\
H9 & 0.3016 & 0.5534 & 0.3296 & $0.050^{*}$ \\
C10 & $0.16807(14)$ & $0.4712(2)$ & $0.23319(12)$ & $0.0407(4)$ \\
C11 & $0.06064(14)$ & $0.3993(2)$ & $0.22426(12)$ & $0.0429(4)$ \\
& & & &
\end{tabular}


supporting information

\begin{tabular}{lllll} 
C12 & $0.02122(14)$ & $0.3625(2)$ & $0.30650(12)$ & $0.0455(4)$ \\
H12 & -0.0499 & 0.3133 & 0.3010 & $0.055^{*}$ \\
C13 & $0.08330(14)$ & $0.3962(2)$ & $0.39585(12)$ & $0.0456(4)$ \\
H13 & 0.0544 & 0.3701 & 0.4497 & $0.055^{*}$ \\
C14 & $0.21523(18)$ & $0.5150(3)$ & $0.14640(13)$ & $0.0576(5)$ \\
H14A & 0.2165 & 0.4083 & 0.1089 & $0.086^{*}$ \\
H14B & 0.2901 & 0.5603 & 0.1659 & $0.086^{*}$ \\
H14C & 0.1693 & 0.6042 & 0.1090 & $0.086^{*}$ \\
C15 & $-0.01190(16)$ & $0.3653(3)$ & $0.12815(13)$ & $0.0599(5)$ \\
H15A & 0.0236 & 0.2788 & 0.0943 & $0.090^{*}$ \\
H15B & -0.0225 & 0.4756 & 0.0927 & $0.090^{*}$ \\
H15C & -0.0832 & 0.3200 & 0.1360 & $0.090^{*}$ \\
\hline
\end{tabular}

Atomic displacement parameters $\left(\AA^{2}\right)$

\begin{tabular}{lllllll}
\hline & $U^{11}$ & $U^{22}$ & $U^{33}$ & $U^{12}$ & $U^{13}$ & $U^{23}$ \\
\hline C11 & $0.0742(4)$ & $0.1002(4)$ & $0.0366(3)$ & $-0.0049(3)$ & $0.0120(2)$ & $0.0047(2)$ \\
O1 & $0.0658(9)$ & $0.0930(10)$ & $0.0483(8)$ & $-0.0276(8)$ & $0.0159(7)$ & $0.0033(7)$ \\
N1 & $0.0472(8)$ & $0.0498(8)$ & $0.0349(8)$ & $-0.0009(6)$ & $0.0069(6)$ & $-0.0023(6)$ \\
C1 & $0.0493(10)$ & $0.0475(10)$ & $0.0428(10)$ & $-0.0042(8)$ & $0.0116(8)$ & $0.0025(7)$ \\
C2 & $0.0427(9)$ & $0.0390(9)$ & $0.0376(9)$ & $0.0015(7)$ & $0.0061(7)$ & $-0.0012(7)$ \\
C3 & $0.0430(10)$ & $0.0469(10)$ & $0.0407(9)$ & $0.0001(7)$ & $0.0093(8)$ & $-0.0005(7)$ \\
C4 & $0.0493(11)$ & $0.0476(10)$ & $0.0364(9)$ & $0.0048(8)$ & $0.0065(8)$ & $0.0002(7)$ \\
C5 & $0.0519(11)$ & $0.0500(10)$ & $0.0432(10)$ & $-0.0005(8)$ & $-0.0013(8)$ & $-0.0027(8)$ \\
C6 & $0.0476(11)$ & $0.0523(11)$ & $0.0550(12)$ & $-0.0103(8)$ & $0.0048(9)$ & $-0.0008(9)$ \\
C7 & $0.0440(10)$ & $0.0450(9)$ & $0.0404(10)$ & $-0.0016(7)$ & $0.0080(8)$ & $-0.0015(7)$ \\
C8 & $0.0421(10)$ & $0.0383(9)$ & $0.0376(9)$ & $0.0011(7)$ & $0.0073(7)$ & $-0.0016(7)$ \\
C9 & $0.0404(9)$ & $0.0421(9)$ & $0.0423(10)$ & $-0.0036(7)$ & $0.0102(7)$ & $-0.0015(7)$ \\
C10 & $0.0480(10)$ & $0.0368(9)$ & $0.0389(9)$ & $0.0015(7)$ & $0.0125(7)$ & $0.0007(7)$ \\
C11 & $0.0482(10)$ & $0.0368(9)$ & $0.0417(10)$ & $0.0013(7)$ & $0.0040(7)$ & $-0.0007(7)$ \\
C12 & $0.0410(9)$ & $0.0474(10)$ & $0.0482(10)$ & $-0.0044(8)$ & $0.0091(8)$ & $0.0017(8)$ \\
C13 & $0.0467(10)$ & $0.0520(10)$ & $0.0400(10)$ & $-0.0016(8)$ & $0.0132(8)$ & $0.0029(8)$ \\
C14 & $0.0672(13)$ & $0.0661(12)$ & $0.0422(10)$ & $-0.0091(9)$ & $0.0171(9)$ & $0.0008(8)$ \\
C15 & $0.0621(12)$ & $0.0674(13)$ & $0.0461(11)$ & $-0.0079(10)$ & $0.0008(9)$ & $-0.0017(9)$ \\
& & & & & & \\
\hline
\end{tabular}

Geometric parameters $\left(\hat{A},{ }^{o}\right)$

\begin{tabular}{llll}
\hline $\mathrm{C} 11-\mathrm{C} 4$ & $1.7365(17)$ & $\mathrm{C} 8-\mathrm{C} 13$ & $1.389(2)$ \\
$\mathrm{O} 1-\mathrm{C} 1$ & $1.336(2)$ & $\mathrm{C} 9-\mathrm{C} 10$ & $1.386(2)$ \\
$\mathrm{O} 1-\mathrm{H} 1$ & 0.8200 & $\mathrm{C} 9-\mathrm{H} 9$ & 0.9300 \\
$\mathrm{~N} 1-\mathrm{C} 7$ & $1.267(2)$ & $\mathrm{C} 10-\mathrm{C} 11$ & $1.396(2)$ \\
$\mathrm{N} 1-\mathrm{C} 8$ & $1.422(2)$ & $\mathrm{C} 10-\mathrm{C} 14$ & $1.505(2)$ \\
$\mathrm{C} 1-\mathrm{C} 6$ & $1.387(2)$ & $\mathrm{C} 11-\mathrm{C} 12$ & $1.385(2)$ \\
$\mathrm{C} 1-\mathrm{C} 2$ & $1.401(2)$ & $\mathrm{C} 11-\mathrm{C} 15$ & $1.500(2)$ \\
$\mathrm{C} 2-\mathrm{C} 3$ & $1.391(2)$ & $\mathrm{C} 12-\mathrm{C} 13$ & $1.372(2)$ \\
$\mathrm{C} 2-\mathrm{C} 7$ & $1.452(2)$ & $\mathrm{C} 12-\mathrm{H} 12$ & 0.9300 \\
$\mathrm{C} 3-\mathrm{C} 4$ & $1.372(2)$ & $\mathrm{C} 13-\mathrm{H} 13$ & 0.9300 \\
$\mathrm{C} 3-\mathrm{H} 3$ & 0.9300 & $\mathrm{C} 14-\mathrm{H} 14 \mathrm{~A}$ & 0.9600
\end{tabular}




\begin{tabular}{|c|c|c|c|}
\hline $\mathrm{C} 4-\mathrm{C} 5$ & $1.375(2)$ & $\mathrm{C} 14-\mathrm{H} 14 \mathrm{~B}$ & 0.9600 \\
\hline $\mathrm{C} 5-\mathrm{C} 6$ & $1.368(2)$ & $\mathrm{C} 14-\mathrm{H} 14 \mathrm{C}$ & 0.9600 \\
\hline $\mathrm{C} 5-\mathrm{H} 5$ & 0.9300 & $\mathrm{C} 15-\mathrm{H} 15 \mathrm{~A}$ & 0.9600 \\
\hline $\mathrm{C} 6-\mathrm{H} 6$ & 0.9300 & C15-H15B & 0.9600 \\
\hline $\mathrm{C} 7-\mathrm{H} 7$ & 0.9300 & $\mathrm{C} 15-\mathrm{H} 15 \mathrm{C}$ & 0.9600 \\
\hline $\mathrm{C} 8-\mathrm{C} 9$ & $1.379(2)$ & & \\
\hline $\mathrm{C} 1-\mathrm{O} 1-\mathrm{H} 1$ & 109.5 & $\mathrm{C} 8-\mathrm{C} 9-\mathrm{H} 9$ & 118.9 \\
\hline $\mathrm{C} 7-\mathrm{N} 1-\mathrm{C} 8$ & $122.85(15)$ & $\mathrm{C} 10-\mathrm{C} 9-\mathrm{H} 9$ & 118.9 \\
\hline $\mathrm{O} 1-\mathrm{C} 1-\mathrm{C} 6$ & $118.44(15)$ & $\mathrm{C} 9-\mathrm{C} 10-\mathrm{C} 11$ & $118.83(15)$ \\
\hline $\mathrm{O} 1-\mathrm{C} 1-\mathrm{C} 2$ & $122.14(15)$ & $\mathrm{C} 9-\mathrm{C} 10-\mathrm{C} 14$ & $120.27(16)$ \\
\hline $\mathrm{C} 6-\mathrm{C} 1-\mathrm{C} 2$ & $119.42(15)$ & $\mathrm{C} 11-\mathrm{C} 10-\mathrm{C} 14$ & $120.89(15)$ \\
\hline $\mathrm{C} 3-\mathrm{C} 2-\mathrm{C} 1$ & $119.12(15)$ & $\mathrm{C} 12-\mathrm{C} 11-\mathrm{C} 10$ & $118.45(15)$ \\
\hline $\mathrm{C} 3-\mathrm{C} 2-\mathrm{C} 7$ & $119.72(15)$ & $\mathrm{C} 12-\mathrm{C} 11-\mathrm{C} 15$ & $120.34(16)$ \\
\hline $\mathrm{C} 1-\mathrm{C} 2-\mathrm{C} 7$ & $121.15(15)$ & $\mathrm{C} 10-\mathrm{C} 11-\mathrm{C} 15$ & $121.20(16)$ \\
\hline $\mathrm{C} 4-\mathrm{C} 3-\mathrm{C} 2$ & $120.07(16)$ & $\mathrm{C} 13-\mathrm{C} 12-\mathrm{C} 11$ & $122.41(16)$ \\
\hline $\mathrm{C} 4-\mathrm{C} 3-\mathrm{H} 3$ & 120.0 & $\mathrm{C} 13-\mathrm{C} 12-\mathrm{H} 12$ & 118.8 \\
\hline $\mathrm{C} 2-\mathrm{C} 3-\mathrm{H} 3$ & 120.0 & $\mathrm{C} 11-\mathrm{C} 12-\mathrm{H} 12$ & 118.8 \\
\hline $\mathrm{C} 3-\mathrm{C} 4-\mathrm{C} 5$ & $120.82(16)$ & $\mathrm{C} 12-\mathrm{C} 13-\mathrm{C} 8$ & $119.34(15)$ \\
\hline $\mathrm{C} 3-\mathrm{C} 4-\mathrm{Cl1}$ & $119.80(14)$ & $\mathrm{C} 12-\mathrm{C} 13-\mathrm{H} 13$ & 120.3 \\
\hline $\mathrm{C} 5-\mathrm{C} 4-\mathrm{Cl} 1$ & $119.37(13)$ & $\mathrm{C} 8-\mathrm{C} 13-\mathrm{H} 13$ & 120.3 \\
\hline $\mathrm{C} 6-\mathrm{C} 5-\mathrm{C} 4$ & $119.86(16)$ & $\mathrm{C} 10-\mathrm{C} 14-\mathrm{H} 14 \mathrm{~A}$ & 109.5 \\
\hline $\mathrm{C} 6-\mathrm{C} 5-\mathrm{H} 5$ & 120.1 & $\mathrm{C} 10-\mathrm{C} 14-\mathrm{H} 14 \mathrm{~B}$ & 109.5 \\
\hline $\mathrm{C} 4-\mathrm{C} 5-\mathrm{H} 5$ & 120.1 & $\mathrm{H} 14 \mathrm{~A}-\mathrm{C} 14-\mathrm{H} 14 \mathrm{~B}$ & 109.5 \\
\hline $\mathrm{C} 5-\mathrm{C} 6-\mathrm{C} 1$ & $120.69(16)$ & $\mathrm{C} 10-\mathrm{C} 14-\mathrm{H} 14 \mathrm{C}$ & 109.5 \\
\hline $\mathrm{C} 5-\mathrm{C} 6-\mathrm{H} 6$ & 119.7 & $\mathrm{H} 14 \mathrm{~A}-\mathrm{C} 14-\mathrm{H} 14 \mathrm{C}$ & 109.5 \\
\hline $\mathrm{C} 1-\mathrm{C} 6-\mathrm{H} 6$ & 119.7 & $\mathrm{H} 14 \mathrm{~B}-\mathrm{C} 14-\mathrm{H} 14 \mathrm{C}$ & 109.5 \\
\hline $\mathrm{N} 1-\mathrm{C} 7-\mathrm{C} 2$ & $121.73(16)$ & $\mathrm{C} 11-\mathrm{C} 15-\mathrm{H} 15 \mathrm{~A}$ & 109.5 \\
\hline $\mathrm{N} 1-\mathrm{C} 7-\mathrm{H} 7$ & 119.1 & $\mathrm{C} 11-\mathrm{C} 15-\mathrm{H} 15 \mathrm{~B}$ & 109.5 \\
\hline $\mathrm{C} 2-\mathrm{C} 7-\mathrm{H} 7$ & 119.1 & $\mathrm{H} 15 \mathrm{~A}-\mathrm{C} 15-\mathrm{H} 15 \mathrm{~B}$ & 109.5 \\
\hline $\mathrm{C} 9-\mathrm{C} 8-\mathrm{C} 13$ & $118.75(15)$ & $\mathrm{C} 11-\mathrm{C} 15-\mathrm{H} 15 \mathrm{C}$ & 109.5 \\
\hline $\mathrm{C} 9-\mathrm{C} 8-\mathrm{N} 1$ & $116.19(14)$ & $\mathrm{H} 15 \mathrm{~A}-\mathrm{C} 15-\mathrm{H} 15 \mathrm{C}$ & 109.5 \\
\hline $\mathrm{C} 13-\mathrm{C} 8-\mathrm{N} 1$ & $125.06(14)$ & $\mathrm{H} 15 \mathrm{~B}-\mathrm{C} 15-\mathrm{H} 15 \mathrm{C}$ & 109.5 \\
\hline $\mathrm{C} 8-\mathrm{C} 9-\mathrm{C} 10$ & $122.23(15)$ & & \\
\hline $\mathrm{O} 1-\mathrm{C} 1-\mathrm{C} 2-\mathrm{C} 3$ & $178.21(15)$ & $\mathrm{C} 7-\mathrm{N} 1-\mathrm{C} 8-\mathrm{C} 9$ & $-178.57(14)$ \\
\hline $\mathrm{C} 6-\mathrm{C} 1-\mathrm{C} 2-\mathrm{C} 3$ & $-1.3(2)$ & $\mathrm{C} 7-\mathrm{N} 1-\mathrm{C} 8-\mathrm{C} 13$ & $1.2(3)$ \\
\hline $\mathrm{O} 1-\mathrm{C} 1-\mathrm{C} 2-\mathrm{C} 7$ & $-0.9(3)$ & $\mathrm{C} 13-\mathrm{C} 8-\mathrm{C} 9-\mathrm{C} 10$ & $0.4(2)$ \\
\hline $\mathrm{C} 6-\mathrm{C} 1-\mathrm{C} 2-\mathrm{C} 7$ & $179.60(15)$ & $\mathrm{N} 1-\mathrm{C} 8-\mathrm{C} 9-\mathrm{C} 10$ & $-179.85(13)$ \\
\hline $\mathrm{C} 1-\mathrm{C} 2-\mathrm{C} 3-\mathrm{C} 4$ & $0.4(2)$ & $\mathrm{C} 8-\mathrm{C} 9-\mathrm{C} 10-\mathrm{C} 11$ & $0.3(2)$ \\
\hline $\mathrm{C} 7-\mathrm{C} 2-\mathrm{C} 3-\mathrm{C} 4$ & $179.50(14)$ & $\mathrm{C} 8-\mathrm{C} 9-\mathrm{C} 10-\mathrm{C} 14$ & $179.07(14)$ \\
\hline $\mathrm{C} 2-\mathrm{C} 3-\mathrm{C} 4-\mathrm{C} 5$ & $0.5(3)$ & $\mathrm{C} 9-\mathrm{C} 10-\mathrm{C} 11-\mathrm{C} 12$ & $-0.8(2)$ \\
\hline $\mathrm{C} 2-\mathrm{C} 3-\mathrm{C} 4-\mathrm{Cl} 1$ & $-178.37(12)$ & $\mathrm{C} 14-\mathrm{C} 10-\mathrm{C} 11-\mathrm{C} 12$ & $-179.62(15)$ \\
\hline $\mathrm{C} 3-\mathrm{C} 4-\mathrm{C} 5-\mathrm{C} 6$ & $-0.4(3)$ & $\mathrm{C} 9-\mathrm{C} 10-\mathrm{C} 11-\mathrm{C} 15$ & $178.03(15)$ \\
\hline $\mathrm{C} 11-\mathrm{C} 4-\mathrm{C} 5-\mathrm{C} 6$ & $178.42(13)$ & $\mathrm{C} 14-\mathrm{C} 10-\mathrm{C} 11-\mathrm{C} 15$ & $-0.7(2)$ \\
\hline $\mathrm{C} 4-\mathrm{C} 5-\mathrm{C} 6-\mathrm{C} 1$ & $-0.5(3)$ & $\mathrm{C} 10-\mathrm{C} 11-\mathrm{C} 12-\mathrm{C} 13$ & $0.8(3)$ \\
\hline $\mathrm{O} 1-\mathrm{C} 1-\mathrm{C} 6-\mathrm{C} 5$ & $-178.16(16)$ & $\mathrm{C} 15-\mathrm{C} 11-\mathrm{C} 12-\mathrm{C} 13$ & $-178.10(15)$ \\
\hline $\mathrm{C} 2-\mathrm{C} 1-\mathrm{C} 6-\mathrm{C} 5$ & $1.4(3)$ & $\mathrm{C} 11-\mathrm{C} 12-\mathrm{C} 13-\mathrm{C} 8$ & $-0.1(3)$ \\
\hline
\end{tabular}


supporting information

$\begin{array}{llll}\mathrm{C} 8-\mathrm{N} 1-\mathrm{C} 7-\mathrm{C} 2 & -179.54(13) & \mathrm{C} 9-\mathrm{C} 8-\mathrm{C} 13-\mathrm{C} 12 & -0.5(2) \\ \mathrm{C} 3-\mathrm{C} 2-\mathrm{C} 7-\mathrm{N} 1 & -178.66(14) & \mathrm{N} 1-\mathrm{C} 8-\mathrm{C} 13-\mathrm{C} 12 & 179.79(15) \\ \mathrm{C} 1-\mathrm{C} 2-\mathrm{C} 7-\mathrm{N} 1 & 0.4(3) & & \end{array}$

Hydrogen-bond geometry $\left(\AA,{ }^{\circ}\right)$

$\mathrm{Cg} 1$ and $\mathrm{Cg} 2$ are the centroids of the $\mathrm{C} 1-\mathrm{C} 6$ and $\mathrm{C} 8-\mathrm{C} 13$ benzene rings, respectively.

\begin{tabular}{lllll}
\hline$D-\mathrm{H} \cdots A$ & $D-\mathrm{H}$ & $\mathrm{H} \cdots A$ & $D \cdots A$ & $D-\mathrm{H} \cdots A$ \\
\hline $\mathrm{O} 1-\mathrm{H} 1 \cdots \mathrm{N} 1$ & 0.82 & 1.87 & $2.5998(19)$ & 149 \\
$\mathrm{C} 3-\mathrm{H} 3 \cdots C g 1^{\mathrm{i}}$ & 0.93 & 2.98 & $3.732(2)$ & 139 \\
$\mathrm{C} 6-\mathrm{H} 6 \cdots C g 2^{\mathrm{ii}}$ & 0.93 & 2.93 & $3.576(2)$ & 128 \\
$\mathrm{C} 14-\mathrm{H} 14 B \cdots C g 2^{\mathrm{iii}}$ & 0.96 & 2.96 & $3.656(2)$ & 131 \\
\hline
\end{tabular}

Symmetry codes: (i) $-x+1 / 2, y-1 / 2,-z+3 / 2$; (ii) $x+1 / 2,-y+3 / 2, z+1 / 2$; (iii) $-x+1 / 2, y+1 / 2,-z+1 / 2$. 